\title{
Dielectric behavior of ceramic-graphene composites around the percolation threshold
}

\author{
Lucía Fernández-García ${ }^{1 *}$, Marta Suárez ${ }^{1}$, José Luis Menéndez ${ }^{1}$, Carlos Pecharromán², Rosa Menéndez ${ }^{3}$ \\ and Ricardo Santamaría ${ }^{3}$
}

\begin{abstract}
$\mathrm{Al}_{2} \mathrm{O}_{3} /$ graphene and $\mathrm{BaTiO}_{3} /$ graphene composites with different concentrations of the conductive second phase, both below and above the percolation threshold, were prepared by the traditional ceramic processing route followed by spark plasma sintering. It is shown that the addition of graphene pins the grain growth of the ceramic matrix grains, leading to a change of the microstructure at low filler concentrations. As a consequence, the composites exhibit two percolation thresholds and their dielectric properties are not only determined by the dielectric properties of the constituents and their relative fractions but also the microstructure of the composite must be considered. Additionally, a giant increase of the dielectric constant has been found around the percolation thresholds in barium titanate-graphene composites. In particular, values of the dielectric constant up to 45,000 and 15,000 were found at $1 \mathrm{kHz}$ in composites containing 0.4 and 0.6 wt. \% graphene, respectively.
\end{abstract}

Keywords: Ceramic composites; Dielectric properties; Percolation threshold

\section{Background}

It is increasingly being recognized that new applications for materials require functions and properties that are not achievable with monolithic materials. In this sense, composites formed by a ceramic matrix and a conductive second phase are very interesting materials since their physical properties, such as optical, electrical, and magnetic properties as well as tribological, corrosion resistance, and wear properties can be tailored, making them attractive for many new applications [1-4]. In particular, the development of ceramic matrix-nanocarbon composites arises as a solution for applications in which materials with an electrical conductivity similar to those of metals and mechanical properties, particularly hardness, are simultaneously required [5].

The dielectric properties of insulator-conductor materials, such as conductivity and permittivity, show a critical behavior when the fraction of the conductive phase reaches the percolation threshold [6,7], which has been the subject of interest of many studies [8-10]. It has

\footnotetext{
* Correspondence: I.fernandez@cinn.es

${ }^{1}$ Centro de Investigación en Nanomateriales y Nanotecnología, Consejo

superior de Investigaciones Científicas (CSIC)—Universidad de Oviedo

(UO)_-Principado de Asturias, Avenida de la Vega 4-6, El Entrego 33940San

Martin del Rey Aurelio (Asturias), Spain

Full list of author information is available at the end of the article
}

been shown both experimentally and theoretically that the percolation threshold strongly depends on the aspect (length-to-diameter) ratio of the conductive particles [11-13]. Hence, it is not surprising that a number of experimental studies have verified the potential of carbon nanotubes as conductive fillers resulting in very low percolation thresholds $[14,15]$. More recently, and due to their extraordinary properties, graphene is being incorporated to new conductor/insulator heterogeneous systems [16, 17]. In this regard, alumina and barium titanate-graphene composites have received a considerable interest in recent times for both their mechanical $[18,19]$ and electrical properties $[20,21]$. In this work, we report how the dielectric properties of ceramic matrix composites are determined by the changes in the microstructure due to the introduction of a conductive second phase of graphene. More specifically, the dielectric properties of spark plasma sintered $\mathrm{Al}_{2} \mathrm{O}_{3}$ /graphene composites with graphene concentrations between 0.56 and $1.24 \%$ weight and $\mathrm{BaTiO}_{3}$ /graphene composites with graphene concentrations between 0.1 and $0.6 \%$ weight are studied in the frequency range of $10^{-1}-10^{6} \mathrm{~Hz}$. 


\section{Methods}

\section{Preparation of Graphene Oxide}

Graphene oxide was synthesized from synthetic graphite by the modified Hummers method [22]. This method employs Hummers reagents with small amounts of $\mathrm{NaNO}_{3}$ and $\mathrm{KMnO}_{4}$. Concentrated $\mathrm{H}_{2} \mathrm{SO}_{4}(360 \mathrm{~mL})$ was added to a mixture of synthetic graphite (7.5 g) and $\mathrm{NaNO}_{3}(7.5 \mathrm{~g})$, and the mixture was cooled down to $0{ }^{\circ} \mathrm{C}$ using an ice bath. Afterwards, $\mathrm{KMnO}_{4}$ (45 g) was slowly introduced in small doses to keep the reaction temperature below $20^{\circ} \mathrm{C}$. The solution was heated to $35{ }^{\circ} \mathrm{C}$ and stirred for $3 \mathrm{~h}$. At that point, a hydrogen peroxide $\left(\mathrm{H}_{2} \mathrm{O}_{2}\right) 3 \%$ solution $(1.5 \mathrm{~L})$ was slowly poured, giving rise to a pronounced exothermal effect up to $98{ }^{\circ} \mathrm{C}$. The reaction mixture was stirred for $30 \mathrm{~min}$ and centrifuged (3700 rpm for $30 \mathrm{~min}$ ) to discard the supernatant. The remaining solid material was then washed with $600 \mathrm{~mL}$ of water and centrifuged again; this process being repeated until the $\mathrm{pH}$ was neutral.

A colloidal suspension of individual graphene oxide sheets in purified water $\left(1 \mathrm{mg} \cdot \mathrm{mL}^{-1}\right)$ was prepared in $1-\mathrm{L}$ batches and kept under ultrasound for $10 \mathrm{~h}$. Afterwards, the suspension was centrifuged ( $3700 \mathrm{rpm}$ for $30 \mathrm{~min}$ ) to discard the filtered supernatant. To prepare the suspensions for organic solvents, water was evaporated in a rotary evaporator and $10 \mathrm{~mL}$ of each solvent was dispersed into $1 \mathrm{mg}$ of solid to then sonicate for $30 \mathrm{~min}$. Details on the characteristics of graphene oxide are given elsewhere [22].

\section{Preparation of $\mathrm{Al}_{2} \mathrm{O}_{3} / \mathrm{Graphene}$ and $\mathrm{BaTiO}_{3} / \mathrm{Graphene}$ Composites}

$\mathrm{Al}_{2} \mathrm{O}_{3}$ /graphene powders with graphene concentrations from 0.56 to $1.24 \%$ weight and $\mathrm{BaTiO}_{3}$ /graphene powders between 0.1 and $0.6 \%$ were prepared as follows: $\mathrm{Al}_{2} \mathrm{O}_{3}$ (Taimei TM-DAR, $>99.99 \%$ purity) and $\mathrm{BaTiO}_{3}$ (Inframat, $>99.95 \%$ purity) powders for ceramic matrices with an average particle size of around 150 and 700 $\mathrm{nm}$, respectively, were dispersed in distilled water then mixed with the graphene oxide dispersion and finally ball milled for $1 \mathrm{~h}$. The homogeneous mixtures were spray dried and uniaxially pressed $(30 \mathrm{MPa})$ prior to spark plasma sintering in an FCT-HP D25/1 apparatus with a heating rate of $50{ }^{\circ} \mathrm{C} \cdot \mathrm{min}^{-1}$ under an applied pressure of $80 \mathrm{MPa}$, a holding time of $1 \mathrm{~min}$ and in vacuum $\left(10^{-1} \mathrm{mbar}\right)$. The final sintering temperature was $1500{ }^{\circ} \mathrm{C}$ in the case of $\mathrm{Al}_{2} \mathrm{O}_{3}$ /graphene composites and $1100{ }^{\circ} \mathrm{C}$ for $\mathrm{BaTiO}_{3} /$ graphene. Densities close to $100 \%$ were found in all composites.

Powder X-ray diffraction analysis (D8 Advance, BRUKER) was used to determine possible high-temperature chemical reactions between components in sintered samples. Raman spectroscopy was performed on a Renishaw 2000 Confocal Raman Microprobe (Rhenishaw Instruments, England) using a 514.5-nm argon ion laser. Spectra were recorded from 1100 to $3500 \mathrm{~cm}^{-1}$. Microstructure was characterized by field emission scanning electron microscopy (FESEM) on a QUANTA FEG 650 and by transmission electron microscopy (TEM) on a JEOLJEM 2100F. The dielectric properties of the samples were studied by standard low-frequency impedance measurements (PSM1735-NumetriQ).

\section{Results and Discussion}

\section{$\mathrm{Al}_{2} \mathrm{O}_{3}$ /Graphene}

$\mathrm{X}$-ray diffraction analysis of $\mathrm{Al}_{2} \mathrm{O}_{3}$ /graphene composites (Fig. 1a) only shows the diffraction peaks corresponding to alumina, indicating that no reaction between the raw powders or decomposition of the matrix has taken place. In order to study the structure of the graphene after sintering, Raman spectra were taken (Fig. 1b). Bands $D$ $\left(1355 \mathrm{~cm}^{-1}\right)$ and $G\left(1583 \mathrm{~cm}^{-1}\right)$ are narrow and present similar intensities $\left(I_{\mathrm{D}} / I_{\mathrm{G}}=0.92\right)$. A shoulder corresponding to the $D^{\prime}$ band $\left(1620 \mathrm{~cm}^{-1}\right)$ is observed next to the $\mathrm{G}$ band and a well-defined structure is also present in the range $2500-3500 \mathrm{~cm}^{-1}$. A single and narrow $2 D$ peak is centered around $2700 \mathrm{~cm}^{-1}$; therefore, according to Ferrari et al. [23], the predominant structure in the composite is one single-graphene layer. A qualitative

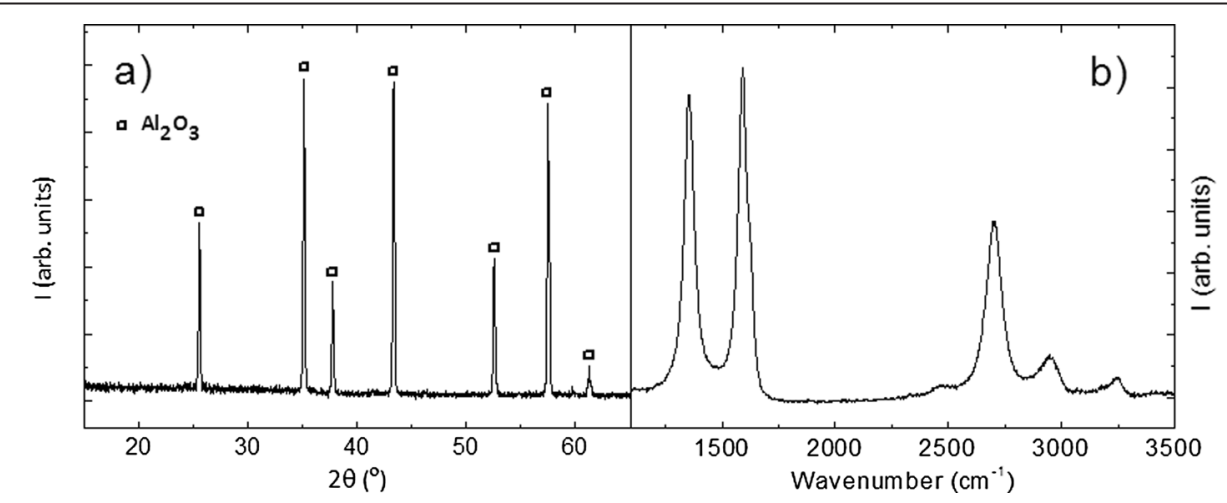

Fig. 1 X-ray diffractogram (a) and Raman spectra (b) for the sample with the highest (1.24\% weight) graphene content 
comparison with the results by Botas et al. [22], considering the relative intensities of the $D$ and $G$ bands together with the shape of the $2 D$ band, indicates that the state of the graphene in these composites is intermediate between those treated at 1000 and $2000{ }^{\circ} \mathrm{C}$ in that work, which agrees with the sintering temperature of $1500{ }^{\circ} \mathrm{C}$.

Electron-dispersive X-ray spectroscopy performed along the yellow line in Fig. 2a on a transmission electron micrograph confirms that graphene agglomerates place on the grain boundaries and triple points. The blue and green lines indicate the presence of aluminum and oxygen, which corresponds to alumina, whereas the red line indicates the presence of carbon. It can be seen that maxima of the red line coincide with positions of minima of the blue and green lines, which happen at the alumina grain boundaries. Fig. $2 \mathrm{~b}$ shows a high-resolution TEM micrograph in which the position of a few stacked graphene layers is indicated by an arrow.

Field emission scanning electron microscopy images (Fig. 3a-d) show differences in the $\mathrm{Al}_{2} \mathrm{O}_{3}$ grain sizes as a function of the graphene concentration in the samples. While monolithic alumina sintered by SPS under the same conditions presents $10-\mu \mathrm{m}$ grain sizes [24], the graphene 0.56 wt. \% sample presented an average alumina grain size around $1 \mu \mathrm{m}$, and while once the graphene content increases, even just to $0.65 \mathrm{wt}$. \%, the alumina grain size decreases to $0.85 \mu \mathrm{m}$. Moreover, for larger graphene contents, the grain size shows a smooth decrease, until it reaches a lower limit around $0.8 \mu \mathrm{m}$. According to Fig. 3e, the grain size reduction is quite relevant, and it indicates that graphene nanosheets act as pinning centers hindering the $\mathrm{Al}_{2} \mathrm{O}_{3}$ grain growth. This result is analogous to the one reported in ref. [24] for alumina-carbon nanofiber composites in which the dielectric properties of the composites were shown to be strongly determined by their topology.

Figure 4 shows the real part of conductivity (Fig. 4a), dielectric constant (Fig. 4b left), and the loss tangent
(Fig. 4b right) of $\mathrm{Al}_{2} \mathrm{O}_{3} /$ graphene composites at a frequency of $1 \mathrm{kHz}$. It should be noted that at low frequencies, the conductivity reaches a plateau regime, as it corresponds to a d.c. regime. In the case of monolithic alumina, its relative dielectric constant and conductivity are respectively $\varepsilon^{\prime}=10$ and $\sigma=10^{-11} \mathrm{~S} \cdot \mathrm{cm}^{-1}$ in agreement with previously reported values in the literature $[25,26]$. For the $0.56 \%$ weight composite, the conductivity increases 4 orders of magnitude while the dielectric constant is multiplied by a factor of 30 , which indicates the proximity of a percolation threshold. However, once the graphene content is raised up to $0.65 \%$, the conductivity decreases two orders of magnitude as well as the dielectric constant decreases to take values close to that of pure alumina. We have attributed this anomalous trend to the drastic changes that ceramic matrix microstructure suffers as shown in Figs. 2-3. In these micrographs, it can be seen that graphene particles are not included into alumina grains. Consequently, the available volume for the conductive phase is restricted to a small layer around the ceramic grain boundaries. In this regard, a reduction of the matrix particle size implies a larger volume to spread, so that the contact or percolation probability between graphene particles reduces in the same manner [27]. In this sense, for higher graphene concentrations $(0.9 \%)$, the conductivity and permittivity increase 8 and 4 orders of magnitude, respectively, indicating that a second percolation threshold has been reached. Higher graphene concentrations lead to minor conductivity increments whereas the dielectric constant takes negative values, which is the expected behavior of metals according to the Drude model [28]. The loss tangent shows a similar behavior to the real part of the dielectric constant.

The existence of a double percolation threshold in ceramic matrix-graphene composites can be very interesting from a technological point of view as it can be used to obtain a giant increase of the dielectric constant in a high permittivity matrix by the addition of very small amounts of graphene as will be shown next.
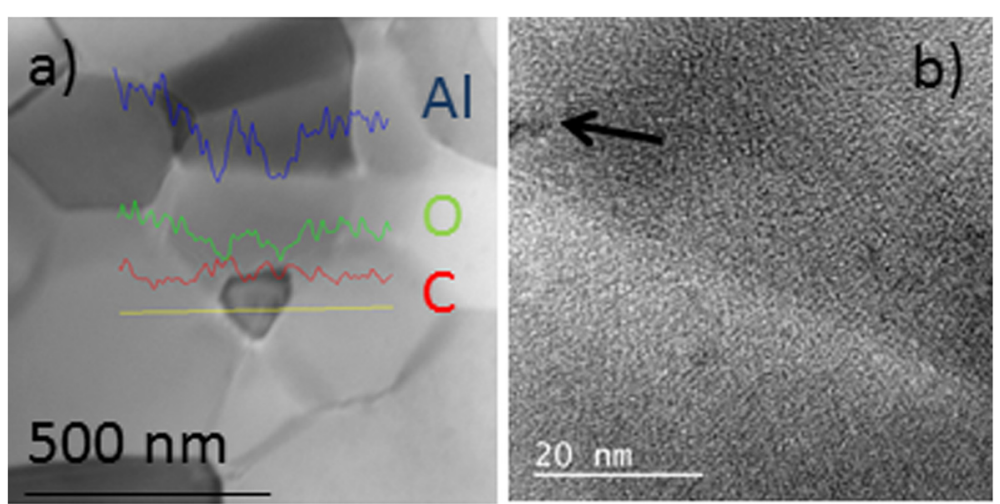

Fig. 2 TEM images of the sample with the highest graphene content (a) with EDX analysis and (b) showing stacked graphene layers 

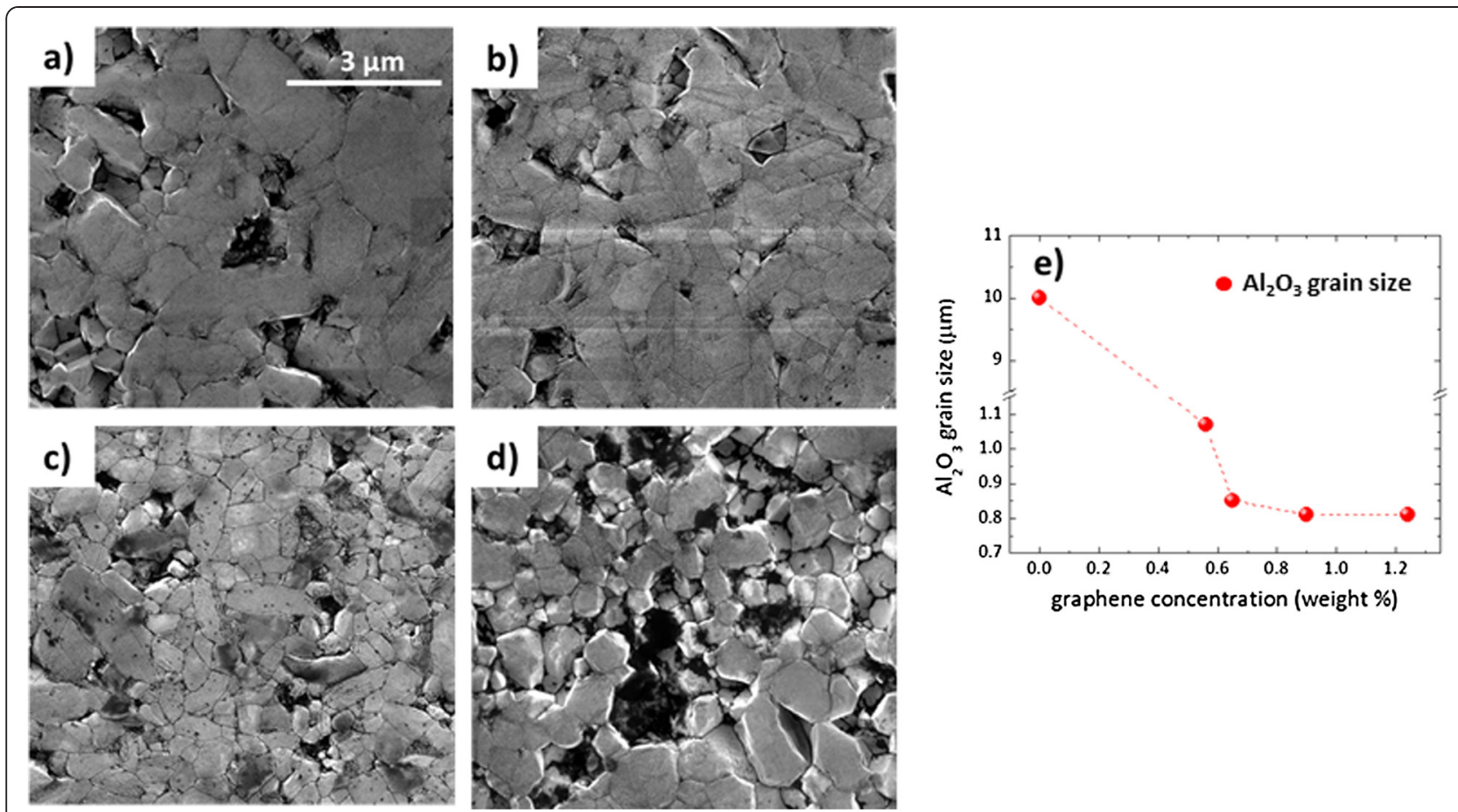

Fig. 3 FESEM images of a 0.56 wt. \%, b 0.65 wt. \%, c 0.9 wt. \%, d 1.24 wt. \% of graphene composites and e alumina grain size vs. graphene weight concentration

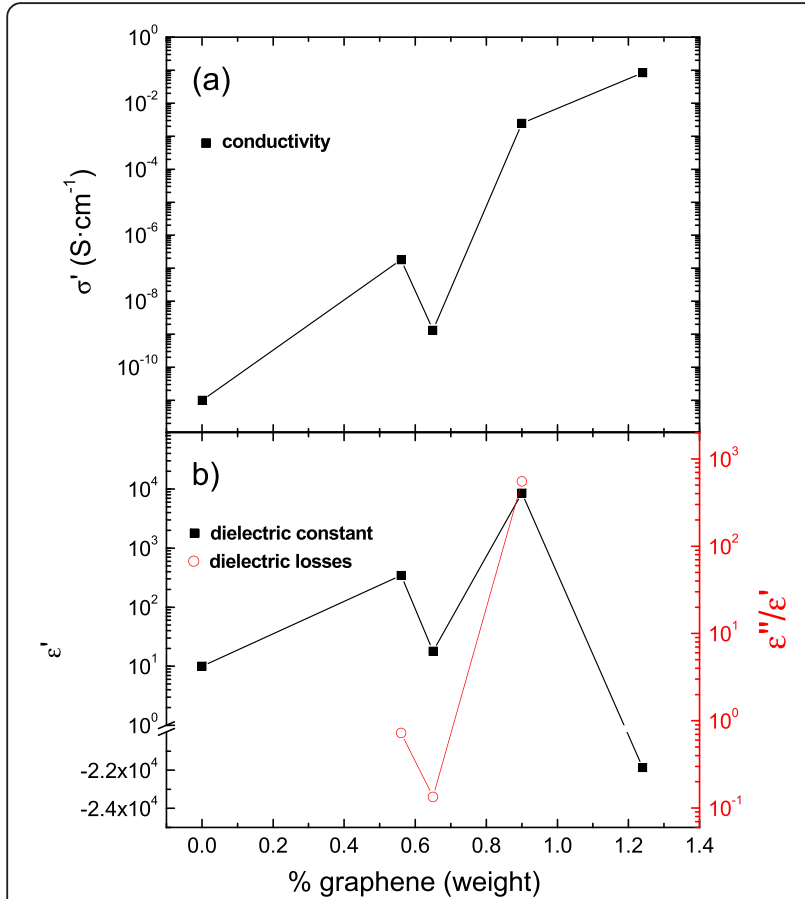

Fig. 4 Real part of a conductivity and $\mathbf{b}$ dielectric constant (real part, left; loss tangent, right) of $\mathrm{Al}_{2} \mathrm{O}_{3}$ /graphene composites vs. graphene weight concentration at a frequency of $1 \mathrm{kHz}$

\section{$\mathrm{BaTiO}_{3} / \mathrm{Graphene}$}

The X-ray diffraction analysis (Fig. 5a) of the sintered samples shows the diffraction peaks corresponding only to the tetragonal phase of $\mathrm{BaTiO}_{3}$. Again, no reaction phases are noticeable in the diffractogram corresponding to the composite with the largest amount of graphene. In this case, this is a very relevant result because some impurities, such as $\mathrm{TiC}$, or different barium oxides could be expected in dense compacts if they were sintered by conventional methods. The use of SPS has allowed obtaining dense composites (theoretical density was around $100 \%$ in all samples) by sintering them at low temperatures and for very short times. In order to study the structure of the graphene after sintering, Raman spectra were taken and are shown in Fig. 5b. Bands $D$ $\left(1355 \mathrm{~cm}^{-1}\right)$ and $G\left(1583 \mathrm{~cm}^{-1}\right)$ are narrow and present similar intensities, with band $D$ being even more intense than band $G(I \mathrm{D} / I G=1.06)$. A qualitative comparison with the results by Botas et al. [22], considering the shapes and relative intensities of the $D, G$, and $2 D$ bands, indicates that the state of the graphene in these composites is very similar to graphene treated at $1000{ }^{\circ} \mathrm{C}$ in the reference given above, which agrees with the sintering temperature of $1100{ }^{\circ} \mathrm{C}$. The $2 D$ peak is centered around $2690 \mathrm{~cm}^{-1}$ and presents a single component; therefore, according to Ferrari et al. [23], the predominant number of stacked layers is one. These results demonstrate that graphite has not been formed. 


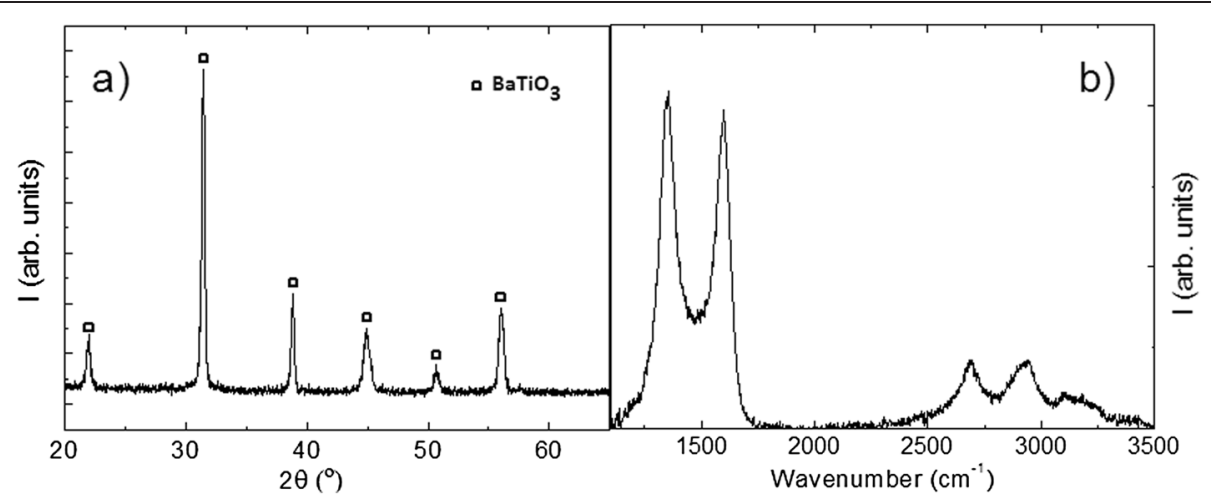

Fig. 5 X-ray diffractogram (a) and Raman spectra (b) for the sample with the highest ( $0.6 \%$ weight) graphene content

The field emission scanning electron microscopy images of $\mathrm{BaTiO}_{3}$ /graphene composites (Fig. 6) show a strong dependence of the average $\mathrm{BaTiO}_{3}$ grain sizes with the graphene concentration in the samples, similar to that shown above for $\mathrm{Al}_{2} \mathrm{O}_{3}$ /graphene composites. In this case, composites up to $0.35 \mathrm{wt}$. $\%$ of graphene have an average $\mathrm{BaTiO}_{3}$ grain size of around $0.9 \mu \mathrm{m}$ (Fig. 6a), while once the graphene content increases above 0.35 wt. \%, the $\mathrm{BaTiO}_{3}$ grain size sharply decreases to 0.55 $\mu \mathrm{m}$ for the 0.45 wt. \% composite (Fig. 6b). For larger graphene contents $(>0.5 \%)$, grain size remains invariable
(Fig. 6c) around $0.5 \mu \mathrm{m}$. Therefore, as in the previous case, the graphene second phase acts as a pinning center hindering the $\mathrm{BaTiO}_{3}$ grain growth.

The dependence of the microstructure versus graphene content is expected to have a deep influence on the dielectric properties of the composites. Fig. 7 shows the real part of conductivity (Fig. 7a), dielectric constant (Fig. 7b, left), and the dielectric loss (Fig. 7b, right) of $\mathrm{BaTiO}_{3} /$ graphene composites at a frequency of $1 \mathrm{kHz}$. Samples with graphene contents up to $0.3 \%$ show similar conductivity values to those of pure $\mathrm{BaTiO}_{3}$, indicating that these
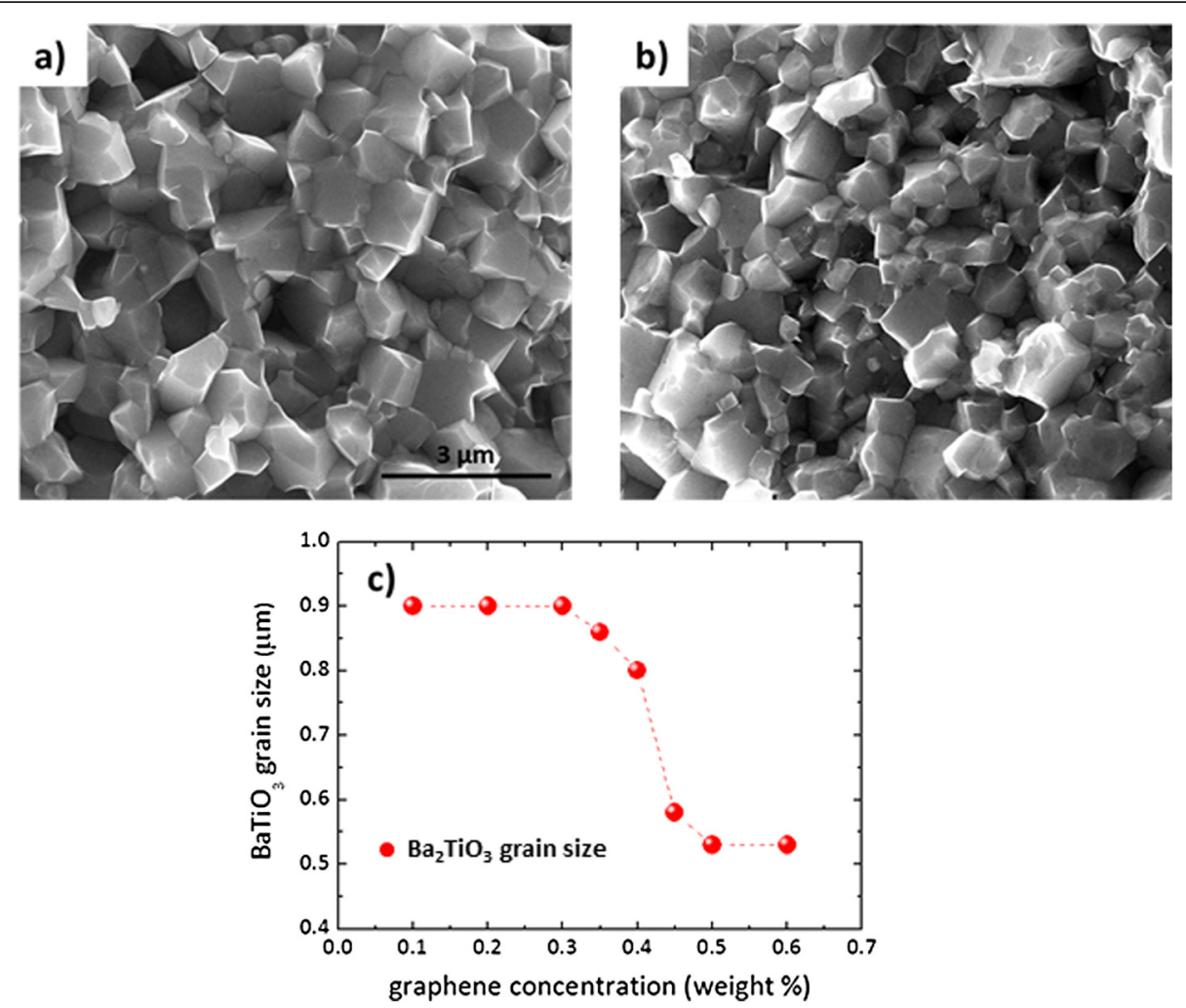

Fig. 6 FESEM images of a 0.1 wt. \%, b 0.45 wt. \% of graphene composites and $\mathbf{c} \mathrm{BaTiO}_{3}$ grain size vs. graphene weight concentration 


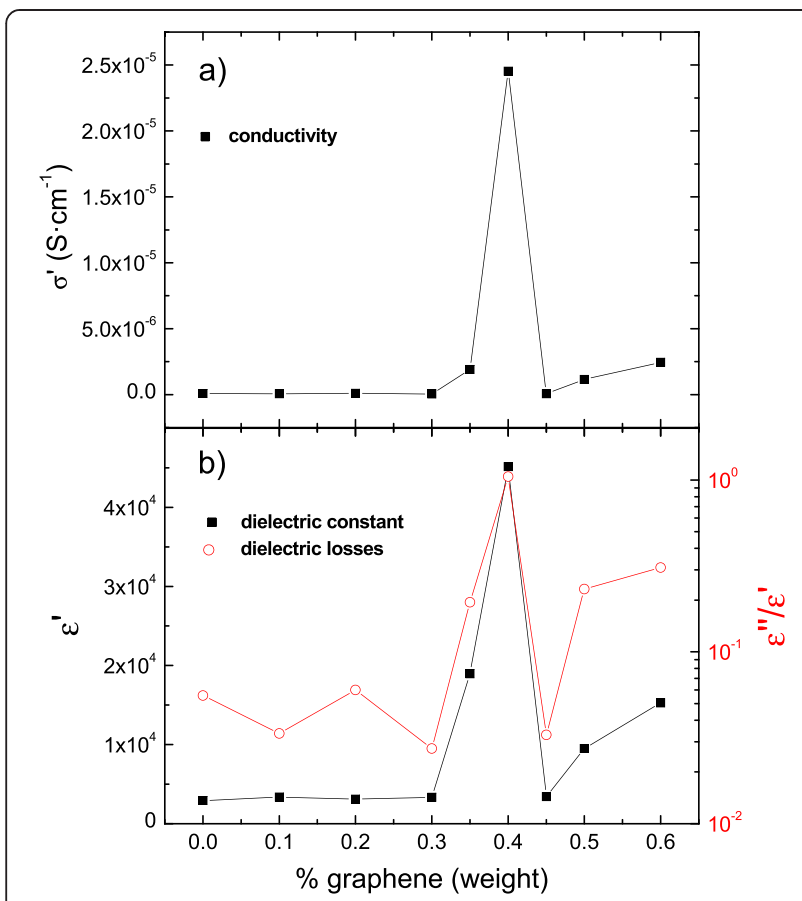

Fig. 7 Real part of $\mathbf{a}$ conductivity and $\mathbf{b}$ dielectric constant (real part, left; loss tangent, right) of $\mathrm{BaTiO}_{3} /$ graphene composites vs. graphene weight concentration at a frequency of $1 \mathrm{kHz}$

composites are below the percolation threshold. In this range of graphene concentrations, the dielectric constant takes the bulk value of pure $\mathrm{BaTiO}_{3}(\sim 2900)$ and the dielectric losses of the composites $(\sim 4-5 \%)$ are due to a reduction of the $\mathrm{Ti}^{4+}$ to $\mathrm{Ti}^{3+}$ originated during the sintering process in vacuum. With increasing graphene content, the conductivity increases until it reaches a maximum value $\left(\sim 2.5 \cdot 10^{-5} \mathrm{~S} \cdot \mathrm{cm}^{-1}\right)$ at $0.4 \%$ graphene. Then, conductivity abruptly decreases for the $0.45 \%$ graphene composite, displaying again a conductivity behavior similar to that of $\mathrm{BaTiO}_{3}$. In a similar way as it happens in the $\mathrm{Al}_{2} \mathrm{O}_{3}$ /graphene composites shown above, the pinning effect on the matrix grains by graphene sheets induces a local decrease (at the grain boundaries) of the effective graphene concentration and, therefore, a decrease in the composite conductivity. Finally, the conductivity of the composites increases again for concentrations over $0.45 \%$.

Analogously to the conductivity, the dependence of the average grain size with the graphene content has a direct influence on the real permittivity values of the composites. For this magnitude, the percolation theory predicts a non-intuitive behavior. According to the work of Efros [6], a near percolated system with conductive inclusions embedded in an insulator matrix should present a sharp maximum of the permittivity. The physical interpretation of this phenomenon can be rationalized as around the percolation threshold, conductor particles are very close between them, but completely isolated by a thin dielectric layer, so that they behave as small capacitors working in parallel; in such manner, the effective capacity is very large. Once the particle concentration exceeds the percolation threshold, particles start touching and the stored charge and capacity sharply diminish. As predicted by the percolation theory of insulator-metal systems [29, 30], a giant increase (over a factor of ten) of the dielectric constant is obtained around the two percolation thresholds. Fig. 7b (left) shows the real part of the dielectric constant of $\mathrm{BaTiO}_{3} /$ graphene composites at a frequency of $1 \mathrm{kHz}$. In particular, the $0.4 \%$ composite exhibits a permittivity value of $\varepsilon^{\prime} \approx 45,000$ and the $0.6 \%$ composite of $\varepsilon^{\prime} \approx$ 15,000 compared to the value of 2900 for the monolithic $\mathrm{BaTiO}_{3}$ ceramic. However, these large values of the dielectric constant are accompanied by large increases of the loss tangent (Fig. 7b, right) contrary to the results by Pecharromán et al. [31]. Given the large aspect ratio and low concentration of the conductive second phase, the control of the local microstructure becomes more difficult, which leads to both locally percolated (responsible of the large dielectric losses) and non-percolated regions.

\section{Conclusions}

In summary, it has been shown that low concentrations of the filler produce a strong change in the microstructure of the ceramic matrix composites, particularly on the average grain size of the ceramic matrix. When the aspect ratio of the conductive filler is large enough, the pinning effect of graphene on ceramic matrix modifies the spatial distribution of the conductive phase in such a manner that the percolation threshold is reached twice as the result of the change of local graphene concentration. The dielectric properties of $\mathrm{Al}_{2} \mathrm{O}_{3} /$ graphene and $\mathrm{BaTiO}_{3} /$ graphene composites at low frequencies depend not only on the graphene concentration but also on the grain size and topology of the composites. This statement has been shown in two different systems, namely, $\mathrm{Al}_{2} \mathrm{O}_{3}$ /graphene and $\mathrm{BaTiO}_{3} /$ graphene. A notable increase (over a factor of ten) of the dielectric constant has been found in $\mathrm{BaTiO}_{3}$ /graphene composites by introducing very small amounts of graphene. In particular, a permittivity value around 45,000 was obtained for the composite of $0.4 \mathrm{wt}$. \% of graphene and around 15,000 for the $0.6 \%$ graphene composite. This opens a route to develop giant dielectric constant composites with very low concentrations of conductive phases.

\section{Competing interests}

The authors declare that they have no competing interests.

\section{Authors' contributions}

The work presented here was performed in collaboration of all authors. RM and RS carried out the preparation of graphene oxide. LFG carried out the synthesis and characterization of the samples and wrote the paper. MS 
participated in the experiments. JLM and CP participated in the design and the discussion of this study and revised the paper. All authors read and approved the final manuscript.

\section{Authors' information}

LFG and MS are PhD degree holders in Chemistry specialized in the fabrication and characterization of ceramic matrix composites. JLM is a tenured scientist and PhD degree holder in Physics specialized in optics and ceramic materials. CP is a senior scientist and PhD degree holder in Physics specialized in the electromagnetic properties of heterogeneous materials. RM and RS are a professor and a senior scientist, respectively, and PhD degree holders specialized in the preparation of carbon precursors and carbon materials from coal and petroleum.

\section{Acknowledgements}

The authors acknowledge funding through projects MAT2011-29174-C02-01 and GRUPIN14-109. L. Fernandez-Garcia acknowledges JAE Predoctoral program for PhD grant. The authors acknowledge support of the publication fee by the CSIC Open Access Publication Support Initiative through its Unit of Information Resources for Research (URICI).

\section{Author details}

${ }^{1}$ Centro de Investigación en Nanomateriales y Nanotecnología, Consejo superior de Investigaciones Científicas (CSIC)—Universidad de Oviedo (UO)_Principado de Asturias, Avenida de la Vega 4-6, El Entrego 33940San Martin del Rey Aurelio (Asturias), Spain. ${ }^{2}$ Instituto de Ciencia de Materiales de Madrid, ICMM-CSIC, Madrid, Spain. ${ }^{3}$ Instituto Nacional del Carbón, INCAR-CSIC, Apartado 73, 33080 Oviedo, Spain.

Received: 5 February 2015 Accepted: 1 May 2015

Published online: 13 May 2015

\section{References}

1. Moya JS, Lopez-Esteban S, Pecharroman C. The challenge of ceramic/metal microcomposites and nanocomposites. Prog Mater Sci. 2007;52:1017.

2. Fahrenholtz WG, Ellerby DT, Loehman RE. $\mathrm{Al}_{2} \mathrm{O}_{3}-\mathrm{Ni}$ Composites with high strength and fracture toughness. J Am Ceram Soc. 2000:83:1279.

3. Tuan WH, Wu HH, Yang TJ. The preparation of $\mathrm{Al}_{2} \mathrm{O}_{3} / \mathrm{Ni}$ composites by a powder coating technique. J Mater Sci. 1995;30:855.

4. Wang X, Padture NP, Tanaka H. Contact-damage-resistant ceramic/single-wall carbon nanotubes and ceramic/graphite composites. Nat Mater. 2004;3:539.

5. Cho J, Boccaccini AR, Shaffer MSP. Ceramic matrix composites containing carbon nanotubes. J Mater Sci. 2009;44:1934.

6. Efros AL, Shklovskii Bl. Critical behaviour of conductivity and dielectric constant near the metal-non-metal transition threshold. Phys Status Solid B. 1976;76:475

7. Nan CW, Shen Y, Ma J. Physical properties of composites near percolation. Annu Rev Mater Res. 2010;40:131.

8. Bauhofer W, Kovacs JZ. A review and analysis of electrical percolation in carbon nanotube polymer composites. Compos Sci Technol. 2009;69:1486.

9. Stauffer D, Aharony A. Introduction to percolation theory. London: Taylor \& Francis; 1992.

10. Sahimi M. Applications of percolation theory. London: Taylor \& Francis; 1994.

11. Celzard A, McRae E, Deleuze C, Dufort M, Furdin G, Marẽché JF. Critical concentration in percolating systems containing a high-aspect-ratio filler. Phys Rev B. 1996:53:6209.

12. Munson-McGee SH. Estimation of the critical concentration in an anisotropic percolation network. Phys Rev B. 1991;43:3331.

13. Qin F, Brosseau C. A review and analysis of microwave absorption in polymer composites filled with carbonaceous particles. J App Phys. 2012;111:061301.

14. Sandler J, Shaffer MSP, Prasse T, Bauhofer W, Schulte K, Windle AH. Development of a dispersion process for carbon nanotubes in an epoxy matrix and the resulting electrical properties. Polymer. 1999:40:5967.

15. Kilbride BE, Coleman JN, Fraysse J, Fournet P, Cadek M, Drury A, et al. Experimental observation of scaling laws for alternating current and direct current conductivity in polymer-carbon nanotube composite thin films. J Appl Phys. 2002;92:4024.

16. He L, Tjong SC. Low percolation threshold of graphene/polymer composites prepared by solvothermal reduction of graphene oxide in the polymer solution. Nano Res Lett. 2013:8:132.
17. Fan P, Wang L, Yang J, Chen F, Zhong M. Graphene/poly(vinylidene fluoride) composites with high dielectric constant and low percolation threshold. Nanotecnology. 2012;23:365702

18. Porwal H, Tatarko P, Grasso S, Khaliq J, Dlouhý I, Reece MJ. Graphene reinforced alumina nanocomposites. Carbon. 2013;64:359.

19. Kim HJ, Lee SM, Oh YS, Yang YH, Lim YS, Yoon DH, et al. Unoxidized graphene/alumina nanocomposite: fracture and wear-resistance effects of graphene on alumina matrix. Sci Rep. 2014;4:5176.

20. Fan YC, Jiang W, Kawasaki A. Highly conductive few-layer graphene/ $/ \mathrm{Al}_{2} \mathrm{O}_{3}$ nanocomposites with tunable charge carrier type. Adv Funct Mater. 2012;22:3882.

21. Wang D, Zhou T, Zha JW, Zhao J, Shi CY, Dang ZM. Functionalized graphene- $\mathrm{BaTiO}_{3} /$ ferroelectric polymer nanodielectric composites with high permittivity, low dielectric loss, and low percolation threshold. J Mater Chem A. 2013;1:6162.

22. Botas C, Alvarez P, Blanco C, Santamaría R, Granda M, Gutierrez MD, et al. Critical temperatures in the synthesis of graphene-like materials by thermal exfoliation-reduction of graphite oxide. Carbon. 2013;52:476.

23. Ferrari AC, Meyer JC, Scardaci V, Casiraghi C, Lazzeri M, Mauri F, et al. Raman spectrum of graphene and graphene layers. Phys Rev Lett. 2006;97:187401.

24. Fernandez-Garcia L, Suárez M, Menéndez JL, Pecharromán C, Nuzhnyy D,

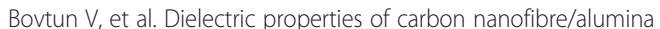
nanocomposites. Carbon. 2013;57:380

25. Chen LY. Electrical performance of co-fired alumina substrates at high temperatures. In: Proceedings of 2012 iMAPS International Conference on High Temperature Electronics. Albuquerque, NM: HiTEC; 2012.

26. Chen LY, Hunter GW. Temperature dependent dielectric properties of polycrystalline $96 \% \mathrm{Al}_{2} \mathrm{O}_{3}$ substrate. In: Proceedings of symposium $\mathrm{G}$ (materials, integration, and packaging issues for high-frequency devices ii), 2004 Mrs Fall Meeting. Boston: Materials Research Society; 2004.

27. Pecharromán C, Iglesias JE. Modeling particle size and clumping effects in the IR absorbance spectra of dilute powders. App Spectrosc. 2000:54:634

28. Drude P. Ueber die reflexion und brechung ebener lichtwellen beim durchgang durch eine mit oberflächenschichten behaftete planparallele platte. Ann Phys. 1891;279:126.

29. Kirkpatrick S. Percolation and conduction. Rev Mod Phys. 1973;45:574.

30. Pecharromán C, Moya JS. Experimental evidence of a giant capacitance in insulator-conductor composites at the percolation threshold. Adv Mater. 2000;12:294.

31. Pecharroman C, Esteban-Betegon F, Bartolome JF, Lopez-Esteban S, Moya JS. New percolative $\mathrm{BaTiO}_{3}-\mathrm{Ni}$ composites with a high and frequency-independent dielectric constant (epsilon( $r$ ) approximate to 80,000). Adv Mater. 2001;13:1541.

\section{Submit your manuscript to a SpringerOpen ${ }^{\circ}$ journal and benefit from:}

- Convenient online submission

- Rigorous peer review

- Immediate publication on acceptance

- Open access: articles freely available online

- High visibility within the field

- Retaining the copyright to your article

Submit your next manuscript at $>$ springeropen.com 